\title{
Video Article \\ Assessing Early Stage Open-Angle Glaucoma in Patients by Isolated-Check Visual Evoked Potential
}

\author{
Xiang Fan $^{1,2}$, Lingling Wu ${ }^{1,2}$, Aihua Ding ${ }^{1,2}$ \\ ${ }^{1}$ Department of Ophthalmology, Peking University Third Hospital \\ ${ }^{2}$ Beijing Key Laboratory of Restoration of Damaged Ocular Nerve, Peking University Third Hospital
}

Correspondence to: Lingling Wu at wullc@hotmail.com

URL: https://www.jove.com/video/60673

DOI: doi:10.3791/60673

Keywords: This Month in JoVE, Issue 159, visual evoked potential, isolated-check, retinal ganglion cell, magnocellular cells, M-cells, pathway, openangle glaucoma, signal-to-noise ratio, cross-sectional study

Date Published: 5/25/2020

Citation: Fan, X., Wu, L., Ding, A. Assessing Early Stage Open-Angle Glaucoma in Patients by Isolated-Check Visual Evoked Potential. J. Vis. Exp. (159), e60673, doi:10.3791/60673 (2020).

\section{Abstract}

Recently, the isolated-check visual evoked potential (icVEP) technique was designed and has been reported to detect glaucomatous damage earlier and faster. It creates low spatial frequency/high temporal frequency bright stimuli and records cortical activity initiated primarily by afferents in the magnocellular ON pathway. This pathway contains neurons with larger volumes and axonal diameters, and it is preferentially damaged in early glaucoma, which can result in visual field loss. The study presented here uses standard operative procedures (SOP) of icVEP to obtain reliable results. It can detect visual function loss using a signal-to-noise ratio (SNR) corresponding to the defects of retinal nerve fiber layer (RNFL) in early stage open-angle glaucoma (OAG). A setting of $10 \mathrm{~Hz}$ and condition of $15 \%$ positive-contrast (bright) are selected to differentiate OAG patients and control subjects, with each check containing eight runs. Each run persists for $2 \mathrm{~s}$ (for 20 total cycles). A flowchart is constructed, which consists of pupil size and intraocular pressure over a $30 \mathrm{~min}$ rest period before each examination. Additionally, the testing order of eyes is performed to obtain reliable electroencephalographic signals. VEPs are recorded and analyzed automatically by software, and SNRs are derived based on a multivariate statistic. An SNR of $\leq 1$ is considered abnormal. A receiver-operating-characteristic (ROC) curve is applied to analyze the accuracy of group classification. Then, the SOP is applied in a cross-sectional study, showing that icVEP can detect glaucomatous visual function abnormality in the central visual field in the form of SNR. This value also correlates with the thickness thinning of RNFL and produces high classification accuracy for early stage OAG. Thus, it serves as a useful and objective diagnostic technology for the early detection of glaucoma.

\section{Video Link}

The video component of this article can be found at https://www.jove.com/video/60673/

\section{Introduction}

Open-angle glaucoma (OAG) is a chronic, irreversible disease and one of the leading causes of blindness. Previous studies have shown that visual field tests, which are the current gold standard for glaucomatous visual loss detection, are based on conventional standard automated perimetry (SAP) cannot detect early glaucomatous functional loss until $20 \%-40 \%$ of retinal ganglion cells (RGCs) are damaged ${ }^{1,2}$. Furthermore, SAP has also been shown to have only moderate test-retest reliability, because it is a subjective psychophysical test and a time-consuming task for patients ${ }^{3}$.

Objective electrophysiological visual field functional measures have better test-retest reliability when detecting glaucoma. Such measures include the multifocal visual evoked potential (mfVEP) and pattern electroretinogram (pERG). However, the pERG cannot provide topographic information, and the mfVEP is more time-consuming than SAP ${ }^{4,5,6,7,8}$. Fortunately, the isolated-check visual evoked potential (icVEP) was recently designed as an additional technique to detect glaucomatous damage earlier and faster ${ }^{9}$.

In the retina, there are several RGC subpopulations such as magnocellular cells (M-cells), parvocellular cells (P-cells), and bistratified cells. They represent parallel pathways for visual information being transmitted to the brain (Figure 1) ${ }^{9,10}$. To govern the separate perceptions of brightness and darkness, the dichotomy of ON and OFF pathways has been established ${ }^{11,12}$. Magnocellular ON (M-ON) cells are considerably larger than magnocellular OFF (M-OFF) cells, while M-cells are considerably larger than P-cells in humans ${ }^{13,14}$. The M-cell pathway mainly conveys low spatial frequency/high temporal frequency information ${ }^{15}$. Thus, cells involved in the M-ON pathway are sensitive to low levels of luminance contrast and not sensitive to chromatic information with larger diameter axons, which are preferentially damaged in early glaucoma ${ }^{16,17}$. Therefore, the icVEP produces low spatial frequency/high temporal frequency bright stimuli and records cortical activity primarily initiated by afferents (such as those found in the M-ON pathway) for the early detection of glaucoma ${ }^{18,19,20,21,22,23}$. 


\section{Protocol}

The study was approved by the Ethics Committee Review Board of Peking University Third Hospital and conformed to the Declaration of Helsinki.

\section{Settings}

NOTE: The icVEP hardware necessitates a reexamination of stimulus conditions for favoring the M-ON pathway using a standard video card with an 8-bit digital-to-analog converter per electron gun.

1. Click the Test CFG button and select icvep-bc-8.cfg.

2. Click the System button, select Configuration $\rightarrow$ Test Configuration and then click the Edit Stimulus button. Please ensure that the frame rate is $60 \mathrm{~Hz}$, luminance of the display's static background is $51 \mathrm{~cd} / \mathrm{m}^{2}$, and total cycles are 20 .

3. To differentiate OAG patients and control subjects, ensure the following conditions: sinusoidal temporal signals of $10 \mathrm{~Hz}(6 \mathrm{frames}$ per cycle) and $15 \%$ positive-contrast (bright, Contrast $7.50 \%$, Luminance Offset $7.50 \%$, Contrast Offset $0.00 \%$ ).

\section{Examination}

1. Select the Eye to Test and ensure that the spatial pattern is a $24 \times 24$ array of isolated-checks to subtend a $11^{\circ}$ visual field, with a $2 \times 2$ array fixation cross without sinusoidal temporal signals, in order to cue facilitated and careful fixation on the center of the screen (Figure 2$)^{9}$.

2. Place the gold cup electrodes filled with electrolytic water-soluble paste at the following midline sites on the scalp based on the international $10-20$ system (Figure 3$)^{24}$. Ensure that the testing distance is $114 \mathrm{~cm}$.

3. Click the Start Test button. One run last for $2 \mathrm{~s}$ : the first second of this period presents half of the test contrast level (7.50\%) as an adaptation condition, and the following second presents the full test contrast $(15.0 \%)$.

4. Note the prompt Error:Outlier from the program and repeat the run when noise is detected and when the electroencephalography (EEG) epoch is rejected.

5. Note the EEG data that is displayed on the operator's monitor when the run is determined to be valid and when the operator is prompted to click Stop Test button in order to reject the data based on reliability.

\section{Automatic data processing using software}

NOTE: The data is calculated by a discrete Fourier transform after EEG signals are recorded.

1. Note that once the data is accepted, the program will instruct the operator by a Ding sound and automatically initiate the next run until a set of 8 valid runs are accumulated.

2. Note that each run produces a fundamental frequency component (FFC), and if one of the FFCs is an outlier relative to the remaining 7, the program will discard that FFC and will prompt the operator to repeat the run until 8 qualified runs are collected.

3. Wait for the program to calculate the mean FFC and radius of a $95 \%$ confidence circle using the $\mathrm{T}^{2}$ circ statistic $^{25}$ that is automatically produced from the 8 FFCs within a few seconds.

4. Ensure that the individual and mean FFC values, confidence circle, and signal-to-noise ratio (SNR) are automatically displayed on the monitor within less than $1 \mathrm{~min}$ after the end of the test (Figure 4A).

\section{Flowchart for assessing reliability of results}

1. Ensure that the refractive error is corrected to adapt for a distance of $114 \mathrm{~cm}$.

2. Ensure that intraocular pressure (IOP) is $\leq 30 \mathrm{mmHg}$ on the day of examination.

3. Ensure that pupil diameters are $\geq 2 \mathrm{~mm}$ and without mydriasis.

4. Ensure that each subject rest and is quiet $\geq 30$ min before examination.

5. To avoid the influence of a study curve, first check the right eye, then left eye; then, check the right and left eyes again, and record this second result.

6. Initiate a retest after at least a 30 min rest when the R-value (nosing ring radius) between both eyes shows a difference of $>0.2$, which means the result is unreliable as mood swings.

\section{Representative Results}

Recent studies showed that the accuracy of icVEP for glaucoma diagnosis ranges from $91 \%-100 \%{ }^{9,22,26}$. Cross-sectional studies in China are presented here to further evaluate the potential diagnostic value of icVEP for early stage OAG. 


\section{Subjects}

Subjects were OAG patients and healthy volunteers recruited by the Department of Ophthalmology, Peking University Third Hospital during 2015 and 2016. Inclusion criteria for OAG patients included the following: 25-75 years of age; best-corrected visual acuity (BCVA) < 0.3 (logarithm of the minimum angle of resolution, log MAR); spherical refraction between -6 and +3 diopters; and transparent ocular media. Additionally, patients showed the presence of OAG (subjects with open-angle, visual field defects corresponding to glaucomatous optic neuropathy [GON], and having normal or elevated IOP without secondary causes), in which the IOP was medically well-controlled and had reliable visual field test results (false positive errors $\leq 20 \%$, false negative errors $\leq 20 \%$, fixation losses $\leq 30 \%$ ) that showed early glaucomatous visual field defects on SAP.

Inclusion criteria for control subjects included the following: no ocular abnormalities, especially no GON in any eye; and a normal IOP that was never elevated over $21 \mathrm{mmHg}$. Exclusion criteria included the following: diabetes or any other systemic disease; history of ocular or neurologic disease; unequal pupil diameters and pupil diameters of $<2.0 \mathrm{~mm}$; poor fixation; current use of medications that can affect visual field sensitivity (i.e., ethambutol, hydroxychloroquine, chlorpromazine); and previous history of intraocular surgery or refractory surgery.

\section{Examinations for OAG diagnosis}

For all patients, spectacle corrections were used to decrease possible effects of a blur on visual field sensitivity. At least two reliable SAP tests were performed by the Humphrey Field Analyzer II 30-2 SITA standard program at baseline. The second reliable visual field result obtained was used in this study to minimize learning effects ${ }^{27}$. An early stage of glaucomatous visual field loss was defined as a mean deviation (MD) of $\geq$ $-6.00 \mathrm{~dB}$, and with at least one of the following: 1) there existed a cluster of $\geq 3$ points in an expected location of the visual field depressed $<$ $5 \%$ level, at least one of which was $<1 \%$ level on pattern deviation plot; 2) corrected pattern standard deviation or pattern standard deviation significant were at $p<0.05 ; 3$ ) glaucoma hemifield test result was "outside normal limits" ${ }^{28}$.

Baseline examination consisted of tests for visual acuity and refraction, pupil-diameter-measurement with a ruler in natural light, slit-lamp biomicroscopy, gonioscopy, Goldmann applanation tonometry (GAT), and dilated stereoscopic fundus examination in all subjects.

The baseline IOP was measured by GAT during glaucoma service (8 A.M. to 11 A.M. local time) on the day after receiving icVEP test reports. Each patient was also subjected to a central corneal thickness (CCT) measurement using ultrasound pachymetry under topical anesthesia ${ }^{29}$. An average of five consecutive readings was recorded.

Stereoscopic fundus photographs were obtained from each patient after pupil dilation and evaluated in a masked fashion by two experienced doctors. Discrepancies between the two doctors were either resolved by consensus or adjudication of a third experienced doctor. GON was defined as at least one of the following: 1) the rim-to-disc ratio was $<0.1$ in the upper or lower rims; 2 ) there existed retinal nerve fiber layer (RNFL) defects; 3) optic disc showed splint hemorrhages ${ }^{30,31}$.

Each patient was also subjected to an optical coherence tomography (OCT) test to confirm RNFL defects corresponding to both stereoscopic photographs and HFA results. The changing of RNFL thickness in temporal superior (TS) quadrant and temporal inferior (TI) quadrant were calculated as follows: changing of RNFL thickness = RNFL thickness value - standard value from database of normal people (Figure 4B).

\section{Statistical analysis}

One eye was randomly selected to be analyzed when both eyes met the inclusion criteria. All the data needed to be established within 3 months for each subject. The SPSS 22.0 statistical package with statistical tests was used as follows: independent sample t-test was used for normally distributed variables; Mann-Whitney $\mathrm{U}$ test was used for numeric variables that were not normally distributed; and binomial variables were compared with a Chi-squared test or Fisher's exact test, when necessary. Receiver-operating-characteristic (ROC) curve analysis was used to estimate prediction accuracy for the presence of glaucomatous damage ${ }^{32}$. Pearson correlation coefficient was used to analyze correlations between SNR and parameters on OCT as well as between SNR and abnormalities in the central $11^{\circ}$ field on SAP. If $p<0.05$, differences were considered significant.

\section{Results}

A total of 44 OAG patients and 39 control subjects were included with complete data. None of these subjects complained during the icVEP test. All 83 subjects were Chinese (48 males and 35 females) with an average age of $48.54 \pm 16.70$ years old (range of 25-74 years). No statistical differences existed in age, sex, right/left eye, BCVA, spherical equivalent, or pupil diameter between patients and controls (Table 1, p $>0.05$ ), but SNR was significantly lower in patients than in controls (Table 1, $p<0.05$ ).

Regarding the icVEP results, there were 30 eyes of early OAG patients that were SNR-positive (68.18\%) and only two eyes in the control group $(5.13 \%)$. Using an SNR criterion of 1, icVEP showed a sensitivity of $68.18 \%$ and specificity of $94.87 \%$ for diagnosing early OAG (calculating an accuracy of $67 / 83[80.72 \%]$ ). However, ROC analysis indicated that an a priori SNR criterion of 0.93 was optimal for discrimination between patients and control subjects (Figure 5). Using an SNR criterion of 0.93 , the specificity of the test reached $100 \%$ with a sensitivity of $65.90 \%$ (calculating an accuracy of $82.10 \%$ ).

For the patients, abnormalities in the central $11^{\circ}$ visual field test (HFA, pattern deviation, central 16 test points; Figure 4C) were calculated by the numbers of abnormal points with different possibility criteria. With a criterion level of $p<0.5$, the amount of abnormal test points in the central $11^{\circ}$ visual field was significantly negatively correlated with SNR $(p<0.05, r=-0.332$, Table 2). Thickness changing of RNFL in the temporal superior quadrant was significantly positively correlated with SNR ( $p<0.05, r=0.370$, Table 2$)$, while SAP-MD, SAP-MD of the other eye, thickness changing of RNFL in the temporal inferior quadrant, and baseline IOP and CCT were all not correlated with SNR $(p>0.05$, Table 3). 


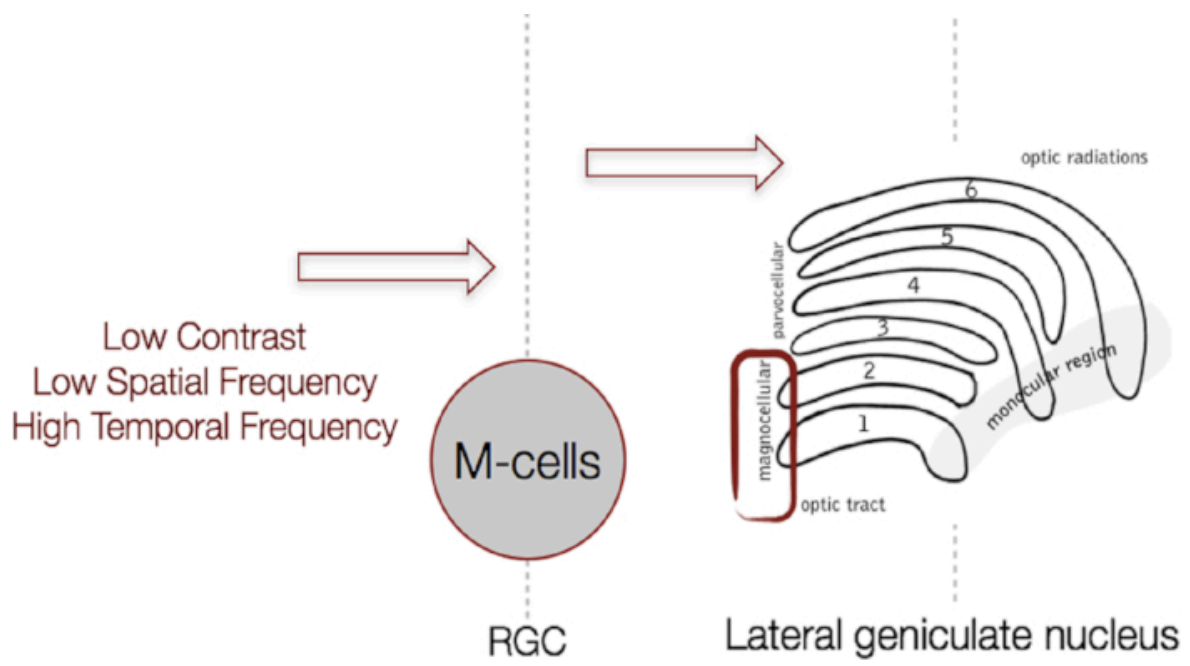

Figure 1: Representation of the isolated-check visual evoked potential evaluating the M-cell pathway. Layers 1 and 2 are involved in the magnocellular pathway. Layers 3, 4, 5, and 6 are involved in the parvocellular pathway. The spaces between these six layers are involved in the bistratified cell pathway. RGC = retinal ganglion cell. Please click here to view a larger version of this figure.

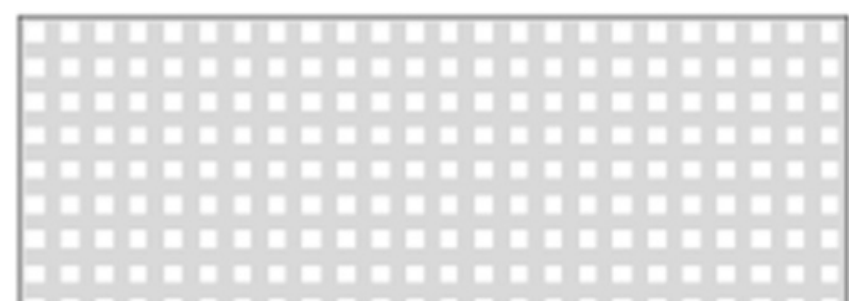

Figure 2: Bright conditions (positive-contrast) on the screen of isolated-check visual evoked potential. This figure has been modified from a previous publication ${ }^{24}$. Please click here to view a larger version of this figure.

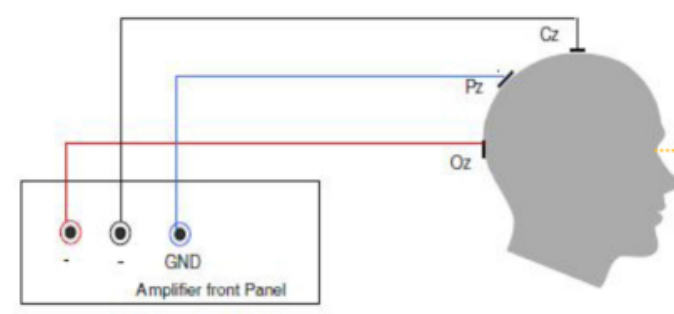

\section{Analysis}

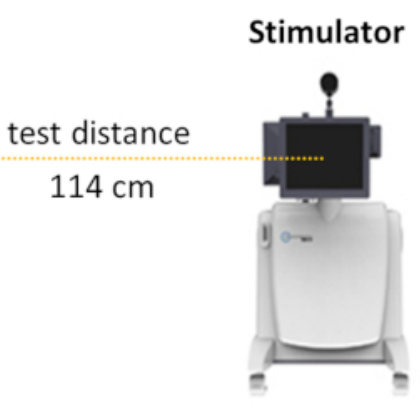

Figure 3: Diagram of isolated-check visual evoked potential examination. $\mathrm{GND}=$ grounding electrode; $\mathrm{Cz}=\mathrm{central}$ midline electrode; $\mathrm{Pz}=$ parietal midline electrode; $\mathrm{Oz}=$ occipital midline electrode. This figure has been modified from a previous publication ${ }^{24}$. Please click here to view a larger version of this figure. 

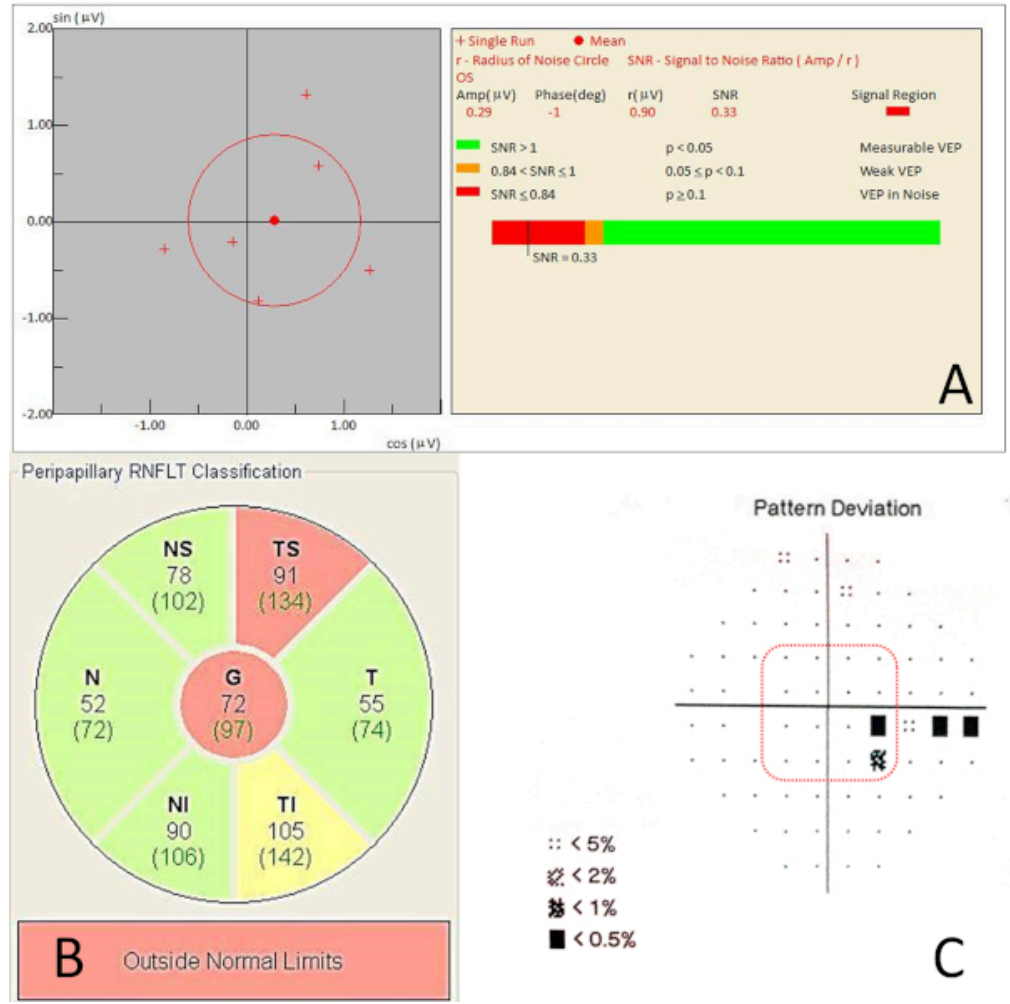

Figure 4: Typical results from an early stage open angle glaucoma patient. (A) Abnormal isolated-check visual evoked potential results. (B) Outcomes of peripapillary retinal nerve fiber layer thickness (RNFLT) classification on the report of optical coherence tomography. Changing of RNFLT = RNFLT value (black number). The standard value from a database of normal subjects. (green number in brackets). $\mathrm{G}=\mathrm{global} ; \mathrm{N}=$ nasal; $\mathrm{T}=$ temporal; $\mathrm{NS}=$ nasal superior; $\mathrm{TS}=$ temporal superior; $\mathrm{NI}=$ nasal inferior; $\mathrm{TI}=$ temporal inferior. (C) Central 16 test points of pattern deviation on Humphrey Field Analyzer 30-2 SITA program corresponding to the central $11^{\circ}$ visual field. This figure has been modified from a previous publication ${ }^{24}$. Please click here to view a larger version of this figure.

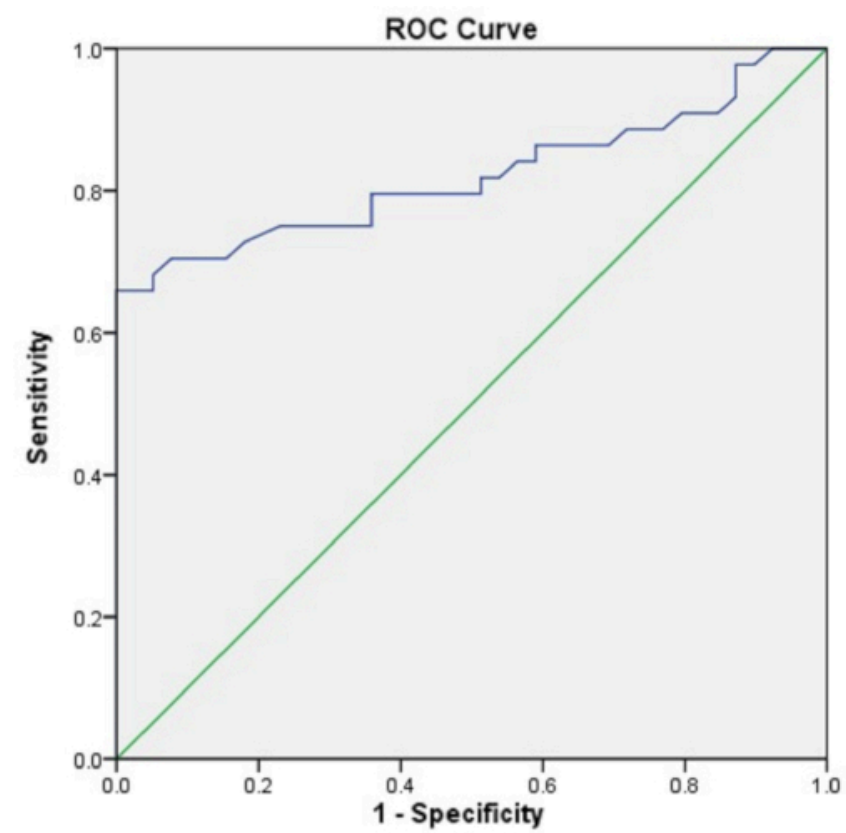

Figure 5: ROC curve. Shown is a ROC curve (blue) for data collected from the signal-to-noise ratios of isolated-check visual evoked potential in open-angle glaucoma patients and control subjects. Please click here to view a larger version of this figure. 


\begin{tabular}{|l|l|l|l|}
\hline & OAG patients $(\mathrm{n}=44)$ & Control subjects $(\mathrm{n}=39)$ & $\mathrm{p}$ \\
\hline Age (year) & $51.59 \pm 14.98$ & $44.72 \pm 16.88$ & $0.053^{*}$ \\
\hline Sex (male/female) & $28 / 16$ & $20 / 19$ & $0.175 \$$ \\
\hline Right eyes / Left eyes & $20 / 24$ & $19 / 20$ & $0.770 \$$ \\
\hline BCVA (log MAR) & $0.04 \pm 0.06$ & $0.01 \pm 0.04$ & $0.093 \#$ \\
\hline Spherical Equivalent (D) & $-1.80 \pm 2.16$ & $-1.30 \pm 2.00$ & $0.276 \#$ \\
\hline Pupil Diameters (mm) & $3.43 \pm 0.50$ & $3.46 \pm 0.51$ & $0.789 \#$ \\
\hline icVEP-SNR & $0.85 \pm 0.53$ & $1.44 \pm 0.57$ & $0.000 \#$ \\
\hline${ }^{*}$ Independent-sample t test, \$Chi-square test, \#Mann-Whitney U test & & \\
\hline $\begin{array}{l}\text { OAG: open-angle glaucoma, BCVA: best-corrected visual acuity; log MAR: logarithm of the minimum angle of resolution; icVEP: isolated-check } \\
\text { visual evoked potential; SNR: signal-to-noise ratio }\end{array}$ & \\
\hline
\end{tabular}

Table 1: Clinical characteristics of OAG patients and control subjects at baseline.

\begin{tabular}{|l|l|l|l|}
\hline Numbers of abnormal test points & Mean \pm Std $(\mathrm{n}=44)$ & $r$ & $\mathrm{p}^{*}$ \\
\hline When $\mathrm{P}<5 \%$ & $4.20 \pm 2.60$ & -0.264 & 0.099 \\
\hline When $\mathrm{P}<2 \%$ & $2.83 \pm 2.34$ & -0.298 & 0.061 \\
\hline When $\mathrm{P}<1 \%$ & $2.08 \pm 2.12$ & -0.266 & 0.097 \\
\hline When $\mathrm{P}<0.5 \%$ & $1.48 \pm 1.80$ & -0.332 & 0.037 \\
\hline${ }^{*}$ Pearson correlation test & & \\
\hline icVEP: isolated-check visual evoked potential; SNR: signal-to-noise ratio; SAP: standard automated perimetry
\end{tabular}

Table 2: Correlation between icVEP-SNR and abnormalities in central $11^{\circ}$ visual field of SAP in open-angle glaucoma patients.

\begin{tabular}{|l|l|l|l|}
\hline & Mean \pm Std $(\mathrm{n}=44)$ & $r$ & $\mathrm{p}^{*}$ \\
\hline SAP-MD $(\mathrm{dB})$ & $-3.83 \pm 1.26$ & 0.115 & 0.457 \\
\hline SAP-MD of the other eye $(\mathrm{dB})$ & $-4.86 \pm 3.94$ & -0.15 & 0.33 \\
\hline $\begin{array}{l}\text { OCT-Thickness changing of RNFL } \\
(\mu \mathrm{m})\end{array}$ & & & \\
\hline Temporal Superior quadrant & $-39.31 \pm 29.89$ & 0.37 & 0.016 \\
\hline Temporal Inferior quadrant & $-43.64 \pm 29.83$ & -0.22 & 0.161 \\
\hline Baseline IOP $(\mathrm{mmHg})$ & $15.48 \pm 2.80$ & -0.121 & 0.435 \\
\hline CCT $(\mu \mathrm{m})$ & $523.24 \pm 29.64$ & 0.171 & 0.333 \\
\hline${ }^{*}$ Pearson correlation test & & & \\
\hline $\begin{array}{l}\text { icVEP: isolated-check visual evoked potential; SNR: signal-to-noise ratio; SAP: standard automated perimetry (HFA 30-2 SITA); MD: mean } \\
\text { deviation; OCT: optical coherence tomography; RNFL: retinal nerve fiber layer; IOP: intraocular pressure; CCT: central corneal thickness }\end{array}$ \\
\hline
\end{tabular}

Table 3: Correlation between icVEP-SNR and relating factors in patients with open-angle glaucoma.

\section{Discussion}

Different settings of icVEP can stimulate different M-cell pathways and create different EEG signals. Under conditions of high temporal frequency $(15 \mathrm{~Hz}$ ) luminance contrast of icVEP (16\% positive-contrast), a study involving 15 OAG patients and 14 normal observers showed a sensitivity of $73.33 \%$ and specificity of $100 \%^{22}$. However, one-half of these patients had advanced OAG. Therefore, for early stage OAG, the sensitivity could not be estimated due to the small sample size.

Tsai's study showed a sensitivity of $78 \%$ (conditions of $15 \%$ positive-contrast and $10 \mathrm{~Hz}$ temporal modulation) and specificity of $100 \%$, with an accuracy of $94 \%$ from the ROC curve. These results improved upon Greenstein's study because of the lower contrast and spatial frequency found in earlier glaucoma patients. Nevertheless, there were less than 11 early stage OAG patients among 18 glaucoma patients (17 openangle, 1 angle-closure) and 16 controls in the study ${ }^{9}$.

In the current study, the OAG patients were only those in early stages and included a much larger sample size, which suggests that icVEP is indeed useful for detecting OAG in the "real" early-stage. About $70 \%$ of early stage OAG eyes were detected by icVEP, and the SNR of patients was greatly different than that of normal subjects.

A recent study showed that pupil size can affect icVEP results in normal subjects. icVEP values were influenced by pupillary constriction and dilation as well as optical blur ${ }^{3}$. This suggests that when obtaining icVEP measurements, the influence of pupil size and optical blur should 
be kept in mind for accurate interpretations. In the current study, pupil size was measured, and it was ensured that all values fell in the normal range. Furthermore, all EEG signals may have been affected by emotions, which yields mostly false positive errors. The current study ensured an IOP of $\leq 30 \mathrm{mmHg}$ on the day of examination to avoid mood swings caused by high pressure. All patients rested for $\geq 30$ min before each examination, and reexamination was also performed to avoid mood effects.

SNR was defined as the ratio of the mean amplitude of the FFC to the radius of the $95 \%$ confidence circle. An SNR of $>1$ indicated a significant response at the 0.05 level, which implied normal electrophysiological activity in the optic nerve. An SNR of $\leq 1$ indicated a response similar to or weaker than the background noise at the 0.05 level, implying abnormal electrophysiological activity in the optic nerve. However, an SNR of 0.93 was optimal for discrimination of early stage OAG patients and control subjects in the current study using a ROC curve. Therefore, a SNR criterion of 0.93 may distinguish the severity of GON in early stage OAG patients for this study.

More than $50 \% \mathrm{M}$ cells were in the macular region; thus, if the fovea was stimulated, there was likely a strong signal resulting in SNR $>1$. Therefore, the $2 \times 2$ array fixation cross on the center of the screen without sinusoidal temporal signals was able to cue-facilitate careful fixation as well as avoid false negative errors with poor fixation ${ }^{34}$. Moreover, recent SD-OCT studies proved that RGCs in the macular region become damaged even in early stages of glaucoma, because proteolysis and secondary axotomy after damage to the optic nerve head may result in RGC apoptosis ${ }^{35,36,37,38}$

Analysis of central 16 test points in the current study based on pattern deviations in HFA corresponded to the $5^{\circ}-10^{\circ}$ of Bjerrum areas, where almost one-half of M-cells are distributed ${ }^{10,11,12,13,14}$. This study showed the numbers of abnormal test points in which different possibility criteria were negatively correlated with SNR (negative R-value); though, only when $p<0.5 \%$ was the correlation significant, suggesting that icVEP was able to detect functional abnormalities and reflect the severity of central visual field loss in early stage OAG.

It has been reported that the responses to stimulation of the P-cell and M-ON pathway are severely disrupted in early stages of glaucoma, even without functional involvement of the central visual field test ${ }^{26}$. However, a limitation of this study is that the icVEP test requires patients with a BCVA value of larger than 0.3 , spherical refraction between -6 and +3 diopters, and transparent ocular media. The study only shows the usefulness of icVEP in early OAG eyes with better visual acuity. Therefore, further studies are needed to create better stimulations and define more accurate criteria for OAG eyes with poorer visual acuity. This will help determine if the icVEP can serve as the optimal functional test for discriminating glaucoma suspects as well as pre-perimetrical and early-stages of OAG. Furthermore, another limitation is that the study does not account for differences between dominant and non-dominant eyes. Differences between those pathways and testing of these two eyes may affect the EEG signals. Above all, the flowchart will be improved after further studies are performed.

In summary, icVEP is able to detect glaucomatous visual function abnormalities in almost $70 \%$ of early stage OAG patients, with a specificity about $95 \%$. The measured functions correlate with both the severity of central $11^{\circ}$ visual field loss of standard automated perimetry and decreases in RNFL thickness as detected by OCT. Therefore, icVEP can serve as a useful and objective electrophysiological visual field functional test for diagnosing early stage OAG.

\section{Disclosures}

All the authors have nothing to disclose.

\section{Acknowledgments}

No funding sources for the work.

\section{References}

1. Quigley, H. A., Dunkelberger, G. R., Green, W. R. Chronic human glaucoma causing selectively greater loss of large optic nerve fibers. Ophthalmology. 95, 357-63 (1988)

2. Quigley, H. A., Dunkelberger, G. R., Green, W. R. Retinal ganglion cell atrophy correlated with automated perimetry in human eyes with glaucoma. American Journal of Ophthalmology. 107, 453-64 (1989).

3. Bjerre, A., Grigg, J. R., Parry, N. R. A. Test-retest variability of multifocal visual evoked potential and SITA standard perimetry in glaucoma. Investigative Ophthalmology \& Visual Science. 45, 4035-40 (2004).

4. Hood, D. C., Zhang, X., Greenstein, V. C. An interocular comparison of the multifocal VEP: a possible technique for detecting local damage to the optic nerve. Investigative Ophthalmology \& Visual Science. 41, 1580-7 (2000).

5. Klistorner, A. I., Graham, S. L. Objective perimetry in glaucoma. Ophthalmology. 107, 2299 (2000).

6. Graham, S. L., Klistorner, A. I., Goldberg, I. Clinical application of objective perimetry using multifocal visual evoked potentials in glaucoma practice. Archives of Opthalmology. 123, 729-39 (2005).

7. Porciatti, V., Ventura, L. M. Normative data for a user-friendly paradigm for pattern electroretinogram recording. Ophthalmology. 111, 161-8 (2004).

8. Ventura, L. M., Porciatti, V., Ishida, K. Pattern electroretinogram abnormality and glaucoma. Ophthalmology. 112, 10-9 (2005).

9. Zemon, V., Tsai, J. C., Forbes, M. Novel electrophysiological instrument for rapid and objective assessment of magnocellular deficits associated with glaucoma. Documenta Ophthalmologica. 117, 233-43 (2008).

10. Gupta, N., Ang, L.-C., de Tilly, L. N. Human glaucoma and neural degeneration in intracranial optic nerve, lateral geniculate nucleus, and visual cortex. British Journal of Ophthalmology. 90, 674-8 (2006).

11. Hartline, H. K. The discharge of impulses in the optic nerve of Pecten in response to illumination of the eye. Journal of Cellular and Comparative Physiology. 2, 465-78 (1938).

12. Schiller, P. H., Sandell, J. H., Maunsell, J. H. R. Functions of the ON and OFF channels of the visual system. Nature. 322, 824-5 (1986). 
13. Kaplan, E., Shapley, R. M. The primate retina contains two types of ganglion cells, with high and low contrast sensitivity. Proceedings of the National Academy of Sciences. 83, 2755-7 (1986).

14. Dacey, D. M., Petersen, M. R. Dendritic field size and morphology of midget and parasol ganglion cells of the human retina. Proceedings of the National Academy of Sciences. 89, 9666-70 (1992).

15. Quigley, H. A., Gregory, R., Dunkelberger, G. R. Chronic human glaucoma causing selectively greater loss of large optic nerve fibers. Ophthalmology. 95, 357-63 (1998).

16. Kerrigan-Baumrind, L. A., Quigley, H. A., Pease, M. E. Number of ganglion cells in glaucoma eyes compared with threshold visual field tests in the same persons. Investigative Ophthalmology \& Visual Science. 41, 741-8 (2000).

17. Xiang, F., Lingling, W., Zhizhong, M., Gege, X., Feng, L. Usefulness of frequency-doubling technology for perimetrically normal eyes of openangle glaucoma patients with unilateral field loss. Ophthalmology. 117 (8), 1530-7 (2010).

18. Zemon, V., Gordon, J., Welch, J. Asymmetries in ON and OFF visual pathways of humans revealed using contrast- evoked cortical potentials. Visual Neuroscience. 1, 145-50 (1988).

19. Zemon, V., Gordon, J. Spatial tuning characteristics of functional subsystems in the visual pathways of humans. Investigative Ophthalmology \& Visual Science. 29 (Suppl), 297 (1988).

20. Zemon, V., Siegfried, J., Gordon, J. Magno and Parvo pathways in humans studied using VEPs to luminance and chromatic contrast. Investigative Ophthalmology \& Visual Science. 32, 1033 (1991).

21. Zemon, V., Eisner, W., Gordon, J. Contrast-dependent responses in the human visual system: childhood through adulthood. International Journal of Neuroscience. 80, 181-201 (1995)

22. Greenstein, V. C., Seliger, S., Zemon, V. Visual evoked potential assessment of the effects of glaucoma on visual subsystems. Vision Research. 38, 1901-11 (1988).

23. Zemon, V., Gordon, J. Luminance contrast mechanisms in humans: visual evoked potentials and a nonlinear model. Vision Research. 46, 4163-80 (2006).

24. Xiang, F., Lingling, W., Xia, D., Tong, D., Aihua, D. Applications of Isolated-Check Visual Evoked Potential in Early Stage of Open-Angle Glaucoma Patients. Chinese Medical Journal. 131 (20), 2439-46 (2018).

25. Victor, J. D., Mast, J. A new statistic for steady-state evoked potentials. Electroencephalography and Clinical Neurophysiology. 78 (5), $378-88$ (1991).

26. Badr, A. A., Zemon, V. M., Greenstein, V. C. M- versus P-function: Relationship to visual field loss in patients with open angle glaucoma. Investigative Ophthalmology \& Visual Science. 44 (13), 50 (2003).

27. Kamantigue, M. E., Joson, P. J., Chen, P. P. Prediction of visual field defects on standard automated perimetry by screening C-20-1 frequency doubling technology perimetry. Journal of Glaucoma. 15, 35-9 (2006).

28. Richard, P. M., Donald, L. B., Paul, P. L. Categorizing the stage of glaucoma from pre-diagnosis to end-stage disease. American Journal of Ophthalmology. 141 (1), 27 (2006)

29. Brandt, J. D., Beiser, J. A., Kass, M. A. Central corneal thickness in the Ocular Hypertension Treatment Study (OHTS). Ophthalmology. 108 (10), 1779-88 (2001).

30. Medeiros, F. A., Sample, P. A., Weinreb, R. N. Frequency doubling technology perimetry abnormalities as predictors of glaucomatous visual field loss. American Journal of Ophthalmology. 137, 863-71 (2004).

31. Yu, Z., Lingling, W., Yanfang, Y. Potential of stratus optical coherence tomography for detecting early glaucoma in perimetrically normal eyes of open-angle glaucoma patients with unilateral visual field loss. Journal of Glaucoma. 19 (1), $61-5$ (2010).

32. Pollack, I., Norman, D. A. A non-parametric analysis of experiments. Psychonomic Science. 1, 125-6 (1964).

33. Salim, S. et al. Influence of pupil size and other test variables on visual function assessment using visual evoked potentials in normal subjects. Documenta Ophthalmologica. 121 (1), 1-7 (2010).

34. Nebbioso, M., Steigerwalt, R. D., Pecori-Giraldi, J., Vingolo, E. M. Multifocal and pattern-reversal visual evoked potentials vs. automated perimetry frequency-doubling technology matrix in optic neuritis. Indian Journal of Ophthalmology. 61 (2), 59-64 (2013).

35. Hood, D. C. et al. Initial arcuate defects within the central 10 degrees in glaucoma. Investigative Ophthalmology \& Visual Science. 52 (2), 940-6 (2011).

36. Hood, D. C. et al. The Nature of Macular Damage in Glaucoma as Revealed by Averaging Optical Coherence Tomography Data. Translational Vision Science \& Technology. 3 (1), (2012).

37. Hood, D. C. et al. Early glaucoma involves both deep local, and shallow widespread, retinal nerve fiber damage of the macular region. Investigative Ophthalmology \& Visual Science. 55 (2), 632-49 (2014).

38. Pescosolido, N. et al. Role of Protease-Inhibitors in Ocular Diseases. Molecules. 19 (12), 20557-20569 (2014). 